\section{Applications of the Helium Ion Microscope}

\author{
Larry Scipioni, Lewis Stern, and John Notte \\ ALIS Business Unit \\ Carl Zeiss SMT, Inc., Peabody, MA \\ l.scipioni@smt.zeiss.com
}

\section{Introduction}

The need for more precise image information of samples coming from fields such as materials analysis, semiconductor processing, and life sciences have pushed the boundaries of charged particle microscopy. A key limitation for the microscope maker in rising to these challenges lies in the relative technical maturity of source technology. Very few changes in sources have occurred in the last generations of tools available to the microscopist, while extensive efforts have been put into reducing aberrations that blur the probe-with their concomitant complexity and cost. Such efforts, representing incremental extensions of these technologies, cannot on their own address many of the emerging needs in nanotechnology. In addition, there are many challenges in the imaging of materials such as polymers and biological specimens that cannot be solved through resolution improvements but rather require different beam sample interaction dynamics. The helium ion microscope (HIM) is a breakthrough technology suited to such challenges.

A previous article has described the source physics and optical characterization of the Atomic Level Ion Source (ALIS) [1]. Based on the field ion microscope with the end form of the tip reshaped by a proprietary technique, it allows essentially all the ion current coming from the tip to originate from only a few atomic sites-thus boosting by orders of magnitude the brightness of the beam from the one site producing ions that are ultimately transported down the column as the probe. A conservative value for the beam brightness puts it in excess of $3.4 \times 10^{9} \mathrm{~A} \mathrm{~cm}^{-2}-\mathrm{sr}^{-1}$.

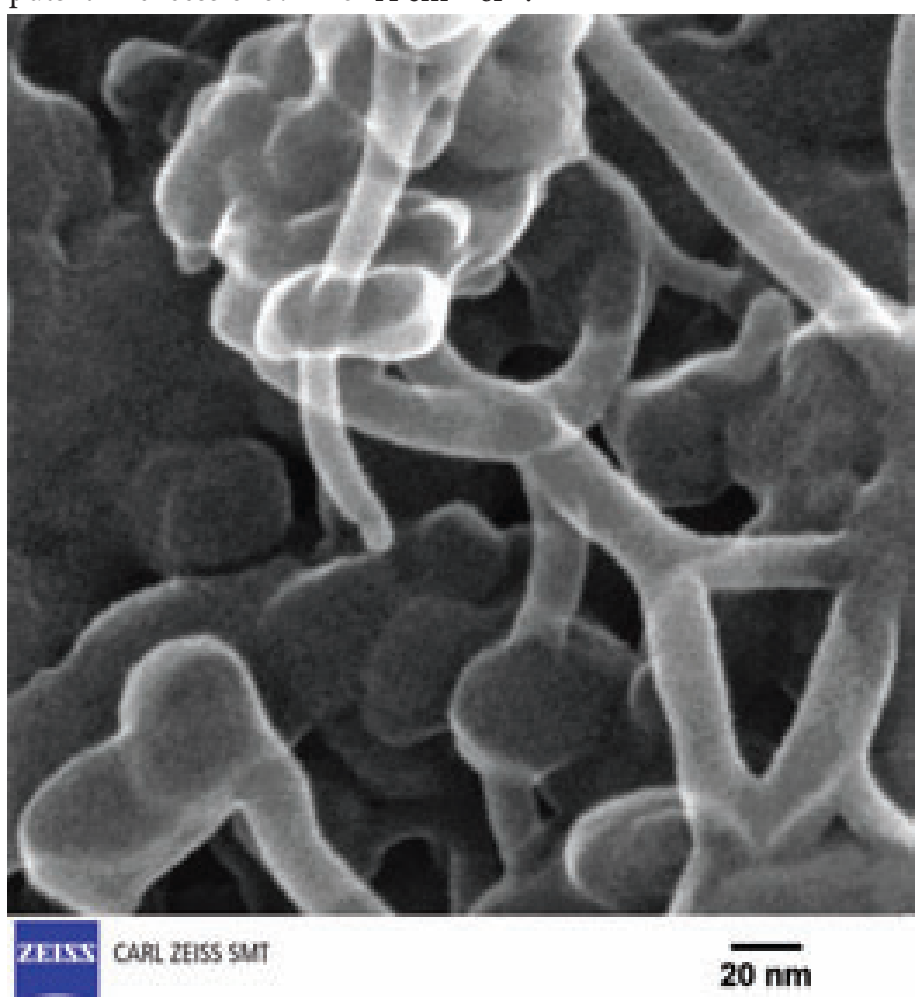

Figure 1: Helium ion micrograph of carbon nanotubes from Orion tool. Beam energy $40 \mathrm{keV}$, secondary electron imaging mode.
There are three factors to consider when weighing the applicability of a technology to imaging: probe size, the interaction volume of the probe with the surface, (which determines the resolution), and the amount of sample information carried with the particles, either secondary or primary, that are emitted from the sample. The ALIS technology offers advantages and new capabilities in these three areas, which are the topic of the remainder of this article.

\section{Line Width Profiles}

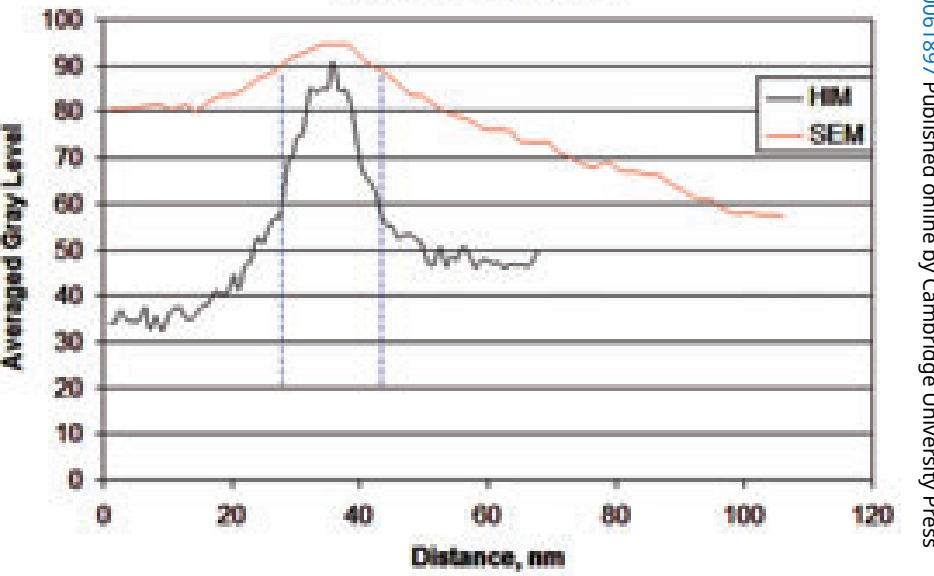

Figure 2: Line width profiles across a thin oxide layer. Each scan is the average of fifty adjacent vertical lines. The dashed lines indicate a rough estimate of the physical width of the feature.

\section{Probe Size}

Image resolution in a scanning microscope is limited in the first instance by the diameter of the best focus of the beam. The ALIS technology employed in the Orion ${ }^{\mathrm{Tm}}$ tool has several advantages in achieving small spot sizes. The small energy spread $(<1 \mathrm{eV})$ and greater operating voltage, compared to a high resolution SEM, significantly reduces the impact of chromatic aberration. Second, since it is an ion source, the diffraction aberration contribution to probe size is reduced by about two orders of magnitude as compared to an electron beam of comparable energy. Third, the extremely small source size $(<1 \AA)$ overcomes the resolution limitation inherent to all other ion sources. This source, combined with the Orion ${ }^{\mathrm{mx}}$ ion optical column, should provide an ultimate practical spot size of about $0.25 \mathrm{~nm}$. Figure 1 shows an example of the progress toward reaching that ultimate resolution. This is a carbon nanotube sample. There are both surface details and sharp edges obvious across this $300 \mathrm{~nm}$ field of view image. A survey of published micrographs show that it is challenging to get SEM images of a carbon nanotube at 50,000× magnification. The HIM image in Figure 1 is at greater than $300,000 \times$. The probe size is estimated to be 0.5 to $0.6 \mathrm{~nm}$. For a nanomaterial characterization application this is critically important, as research looking into the properties of these objects will need to determine the way in which they interface to their surroundings-a critical link to behavior. Our future work will investigate devices that incorporate materials such as these nanotubes in order to create function.

\section{Beam-Surface Interaction Volume}

We almost cannot over-emphasize the importance of the dynamics of beam-sample interactions in achieving the best results. If the secondary particles defining the image pixels are not local to the probe landing area, then the resolution is degraded. In SEM for example, backscattered electrons create type II secondary electrons at surface locations outside the probe landing area. It is also these 


\section{Beating the Competition is Easy, When You've Got Products They Don't Offer!}

Si-Li EDS Detectors,

IXRF offers guaranteed, premium $\leq \underline{130 \mathrm{eV}}$, resolution Si-Li detectors. (*industry standard detector resolution $133-138 \mathrm{eV}$ ) that carry a three year warranty.

New $30 \mathrm{~mm}^{2}$ at $133 \mathrm{eV}$, three year warranty available for cost effective fast X-ray Mapping.

\section{fX SEM XRF,}
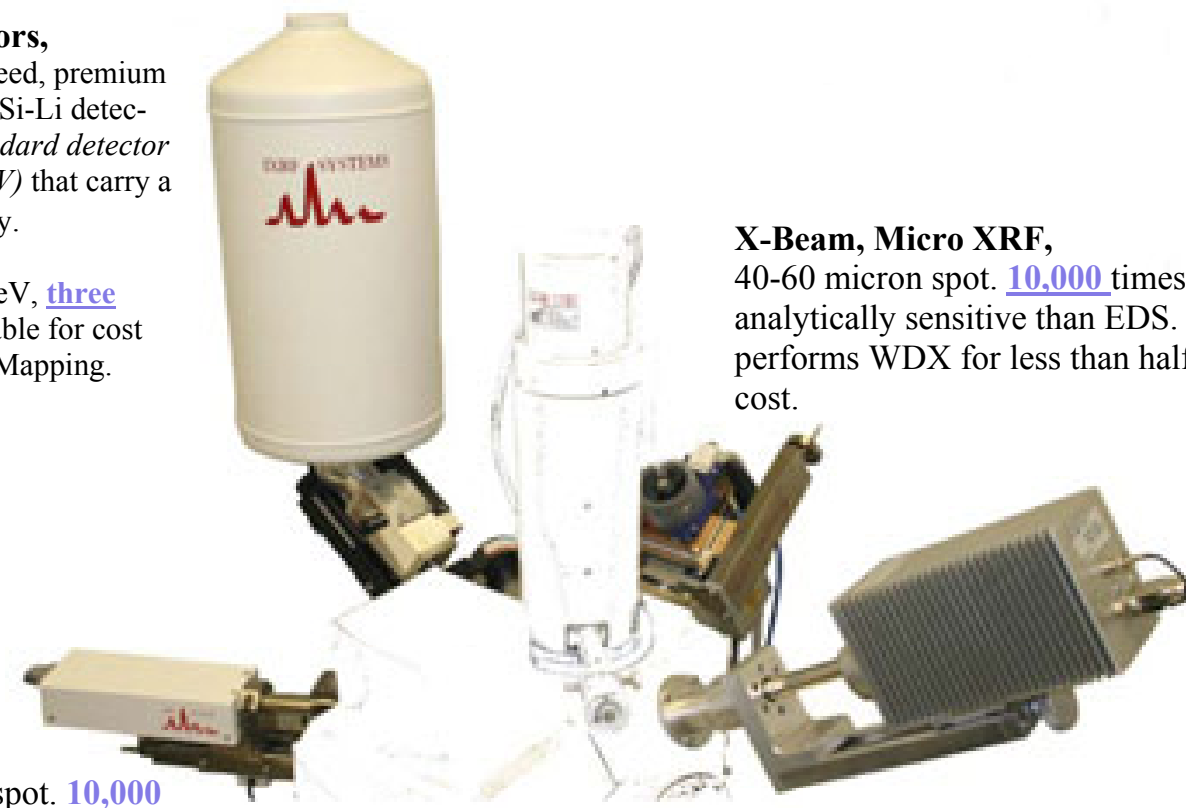

times more analytically sensitive than EDS.

Perfect for environmental applications as well as non-conductive samples.

50 $\mathbf{m m}^{2}$ Silicon Drift Detector, the largest Active Area in the industry $\left(50 \mathrm{~mm}^{2}\right) .133 \mathrm{eV}$ resolution, with optimum peak stability, and high input count rates. Who says "Bigger isn't better"?

IXRF does not stratify their software suite into low, medium, and high-end levels; there's only one high-end package that includes a myriad of Spectra Analysis, Image Analysis, X-ray Mapping, and unsurpassed SEM/EDS Automation.

Free Software Upgrades for Life...no compromises.

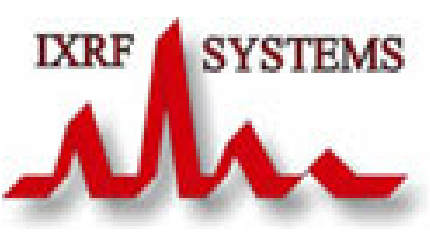

IXRF Systems, Inc. 15715 Brookford Dr, Houston, Texas, 77059 (Tel): 281/286-6485 (Fax): 281/286-2660 www.ixrfsystems.com 
higher energy electrons, which give the appearance of transparency to nano-dimensioned samples, for backscattered particles from rear surfaces, or background objects that penetrate back to the top surface and are detected. Likewise, the resolution of EDS mapping with SEM is limited to about $1 \mu \mathrm{m}$ due to the large $\mathrm{x}$-ray generation volume, even though the probe is much smaller. Helium ions penetrate deeply into the surface and produce no high energy electrons, so the interaction volume is defined very well by the beam width and the escape depth of low energy secondary electrons (a few nanometers). Looking again at Figure 1, we can see that the transparency of these nanotubes is only moderate. Several junctions between tubes can be observed easily, and the surface topography of the nanotubes becomes visible. The scattering of SEM electrons laterally from the sides of physical edges also creates a signal peak that registers as a false flaring in the image around the edge of three dimensional features. This effect is nominal with the helium ion probe, as evidenced in Figure 1. For measurements such as semiconductor metrology this becomes critical. An example of how these effects impact measurements is the imaging of cross sections of thin layers-where false information on the edge transitions can overwhelm the actual layer data desired, is seen more quantitatively in Figure 2, which contains line width profiles from both HIM and SEM images extracted from an oxide layer in a semiconductor device. The device was cleaved to present a cross section to the beam, and each profile is the average of 50 adjacent line scans taken perpendicular to the layer. We note that the position of the edge is easily identified in the HIM image, whereas the SEM derived profile is of limited use due to insufficient contrast. Thus we conclude that helium ion microscopy will find many applications in metrology and defect review studies.

\section{Materials Related Contrast}

Helium ion induced secondary electron yields vary according to material more than is seen with a primary electron beam. This provides higher material-related contrast. It is possible to improve this contrast even more if we image with the backscattered ions.

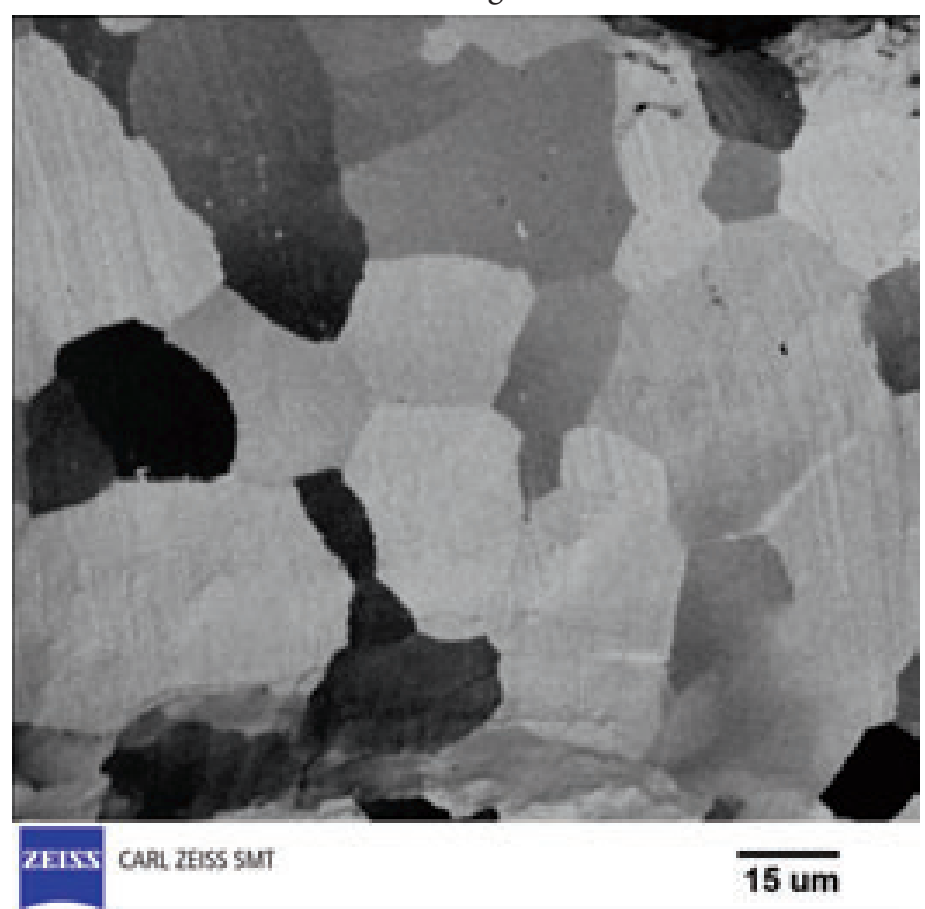

Figure 3: Helium ion micrograph displaying channeling contrast. The imaging was obtained using backscattered helium ions. The grains in the image were created by crimping a single crystal metal sample.
These particles convey material information due to a Rutherfordlike dependence of the total backscattering probability on the target atomic number. There is also the ability to gain crystal orientation information through channeling, wherein the backscattering probability changes according to the orientation of the crystal planes with respect to the beam axis. Figure 3 shows the formation of grains in a single crystal due to mechanical stress, in this case by crimping off a piece of single crystal wire.

Spread of Transmitted lons

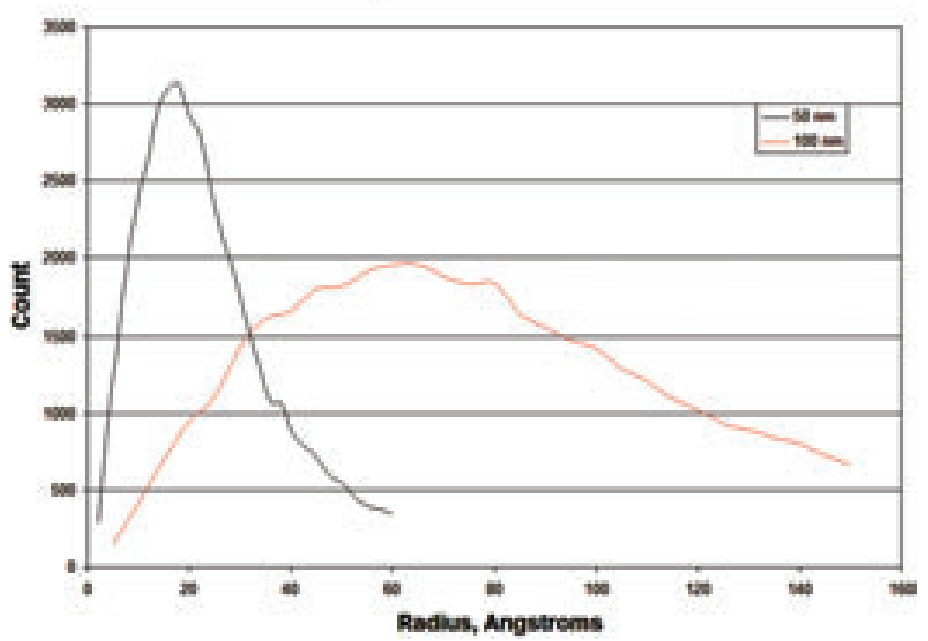

Figure 4: Simulated spread for a $40 \mathrm{keV}$ helium ion beam passing through 50 and $100 \mathrm{~nm}$ thick layers of Silicon.

As with electron backscatter imaging, the total abundance highlights material changes qualitatively, but it does not allow for unique elemental identification. In high energy Rutherford backscattering spectroscopy, the energy and angle dependence of backscattered particles is used to analyze the sample. We are exploring ways to capture this information as a means to add compositional information to the HIM.

For a final note, this mode facilitates imaging of non-conducting samples, since we are detecting high energy particles whose trajectories are not perturbed by sample potential variations such as might occur under the beam. The Orion ${ }^{\text {tw }}$ tool is also equipped with a low energy electron flood gun for instances where we desire to image via secondary electrons. This gun can provide free electrons once per scan line or once per image frame in order to control the sample surface potential. Without this, most of the material contrast is lost.

\section{Novel Operating Modes}

There are unique applications arising out of the microscope due to the nature of the interaction of the helium ions with the samples. One possible imaging mode that might hold much promise is imaging based upon ions transmitted through a thin sample. Indeed, proof of principle work at an energy similar to the HIM microscope was carried out more than twenty years ago with hydrogen by Escovitz et. al. [2]. Dubbed STIM (scanning transmission ion microscopy), this technique has been applied with protons of a few $\mathrm{MeV}$ energy to study topics such as crystal channeling [3] and biological tissues [4]. This technique requires light ions $(\mathrm{H}$ or $\mathrm{He})$ in order to get sufficient transmission. What is novel with the application of the ALIS source is the possibility of looking at thin samples under a probe with dimensions similar to atomic nearest neighbor distances and the possibility to obviate the need for high energy particle sources. ALIS-STIM promises to provide new sources of image information. 


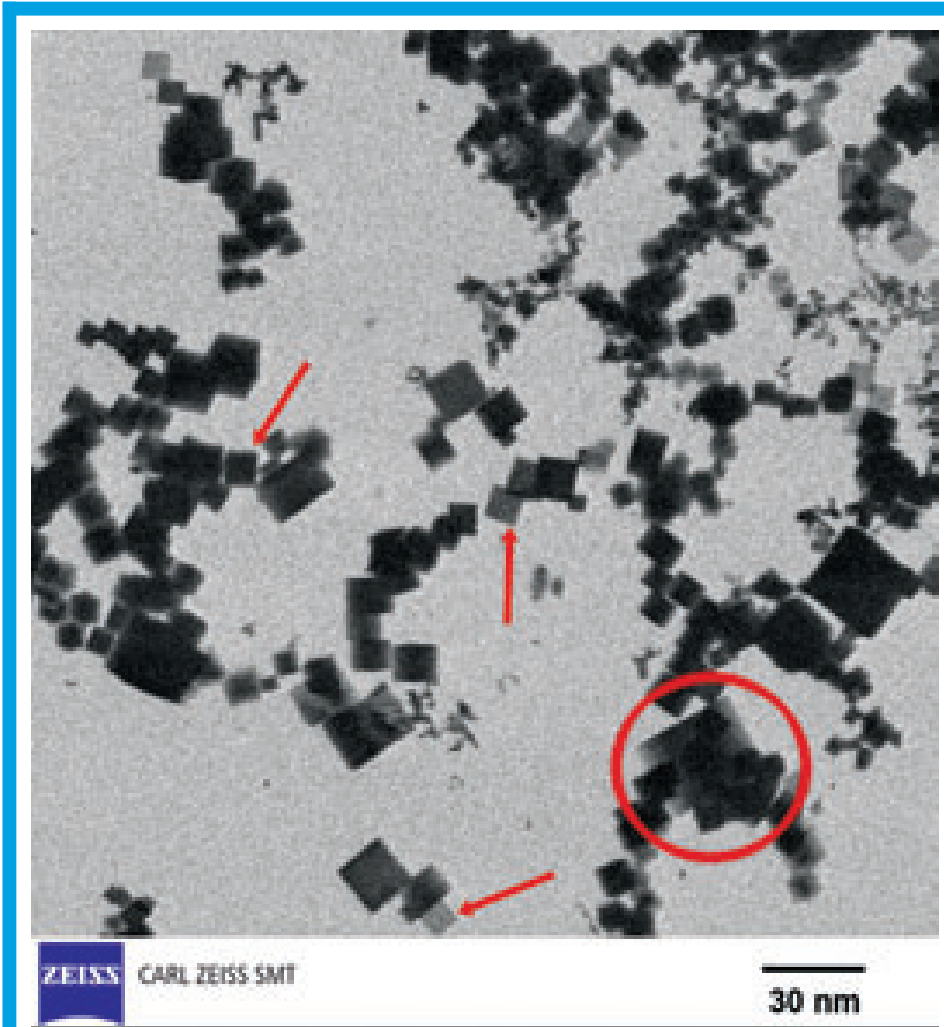

Figure 5: Scanning Transmission Ion Micrograph of magnesium oxide particles on a carbon film. Arrows indicate crystals of similar size but differing contrast levels, while the circle surrounds a cube facing the beam edge on.

A critical issue to consider in such an application is the spreading of the beam due to scattering in the material. There needs to be some interactions in order to gain image information, but we cannot have excessive spreading or beam energy loss. These would eliminate the high resolution information; energy loss and, in addition, may damage the specimen to some extent.

To consider this issue, we have run TRIM [5] simulations of $40 \mathrm{keV}$ helium ions passing through thin silicon samples. In each case 50,000 trajectories were run to find the spectrum of transmitted ions. As all incoming ions are simulated originating at the same position, the plot of the radial distance of transmitted ions, shown in Figure 4, indicates the delocalization the beam suffers. For a 50 $\mathrm{nm}$ thick Si sample, $50 \%$ of a point beam remains within $2.2 \mathrm{~nm}$ of the impact axis. Once the sample thickness exceeds about $80 \mathrm{~nm}$, however, the amount of spreading begins to rise rapidly. We must note at this point that TRIM does not take channeling effects into account. This phenomenon will maintain beam collimation much better, enhancing grain contrast. The HIM image in Figure 5 emphasizes this by showing a STIM image of MgO crystals. The arrows overlaying the image locate crystals (which are cubes) of similar size but having much different contrast levels, indicative of changes in channeling, which is in turn indicative of crystal orientation. The circle over the larger crystal highlights a cube with its edge facing the beam, so that the integrated thickness is less both on the left and the right-thus it appears lighter at those extremities. We intend to investigate channeling between atomic planes of the ultimate $2.5 \AA$ diameter beam as a means of lattice imaging with ions. For the 50 $\mathrm{nm}$ simulated sample, TRIM also indicates that $99.7 \%$ of the ions are transmitted, with an average energy loss per ion of $6100 \mathrm{eV}$. Damage will be greatly reduced as compared to a bulk sample (7.2 vacancies/ ion as opposed to 156), and there is essentially no implantation. Thus this mode could be beneficial for sensitive samples.

We realize also that there are other possible ion-sample interactions that will support unique imaging applications. As an example, consider the image of common salt crystals in Figure 6, taken with the helium ion beam. We were looking in this case for visible light by collecting only photons directly into a photomultiplier tube light pipe. The photons generated may arise either from excitations induced in the crystal or from the neutralization of the helium ions. Such an imaging mode could be used to locate fluorescent markers on biological samples, to study photoluminescence in nanostructures, or to detect buried materials. This ionoluminescence provides intriguing evidence of further valuable analytical abilities of the HIM technology.

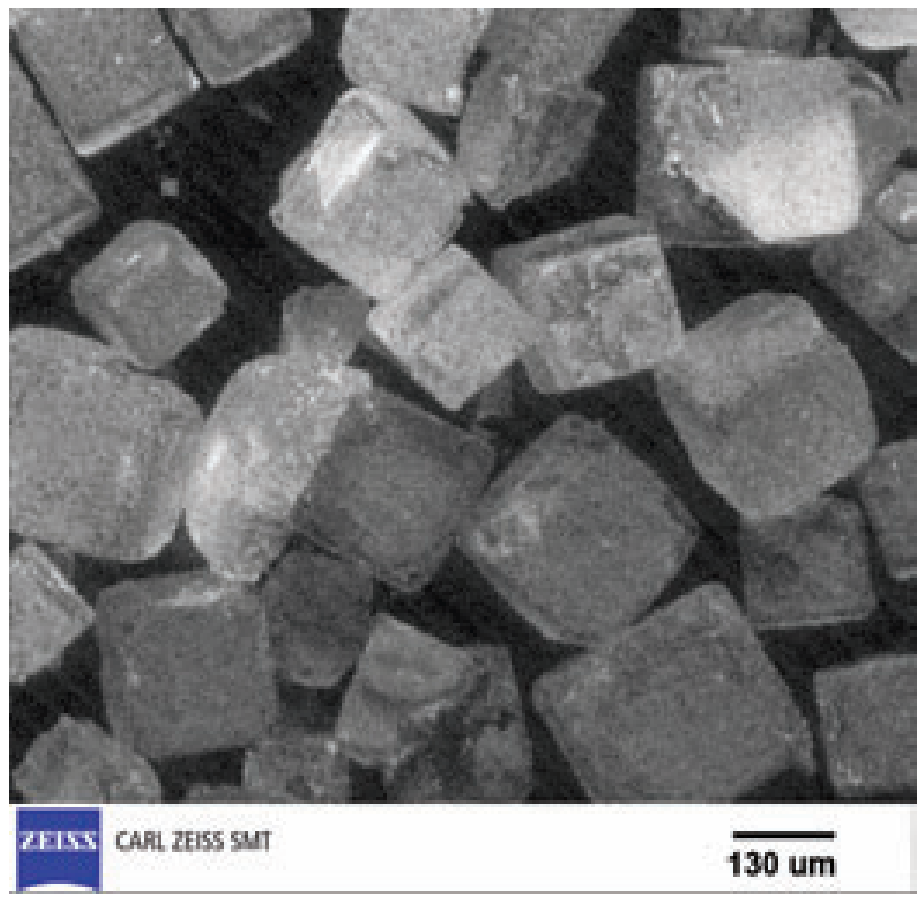

Figure 6: Helium ion micrograph taken with detection of ion induced photon emission. A photomultiplier was used to collect visible band light from this sample of salt $(\mathrm{NaCl})$. Field of view is $1350 \mu \mathrm{m}$.

\section{Conclusion}

The development of the Orion ${ }^{\mathrm{TM}}$ helium ion microscope is now proving applications in many areas. Sub-nanometer probe sizes have been measured, with further improvements expected. Perhaps just as important, the new beam-sample interaction dynamics seen with the helium ion beam as compared to SEM allows better extraction of information from the images. There are also opportunities to develop new imaging modes that hold promise of revealing additional sample properties.

\section{References}

[1] J. Morgan, J. Notte, R. Hill, B. Ward: An Introduction to the Helium Ion Microscope, Microscopy Today 16 No. 4, p. 24 (2006).

[2] W. H. Escovitz, T. R. Fox, And R. Levi-Setti: Scanning Transmission Ion Microscope with a Field Ion Source, Proc. Nat. Acad. Sci. 72, No. 5, pp. 1826-1828 (1975)

[3] M. Cholewa, G. Bench, G. J. F. Legge, A. Saint: Appl. Phys. Lett. $\underline{56}$, Iss 13, pp. 1236-1238 (1990).

[4] T. Reinert; A. Sakellariou; M. Schwertner; J. Vogt; T. Butz: Nucl. Instr. Meth. Phys. Res. B 190, No. 1, pp. 266-270 (2002).

[5] Calculation done with SRIM-2006.02 @ 